\title{
Energetics of carbon nanotubes with open edges: Modeling and experiment
}

\author{
V. G. Zavodinsky ${ }^{1}$, O. A. Gorkusha ${ }^{2}$, A. P. Kuz'menko ${ }^{3}$ \\ ${ }^{1}$ Institute for Material Science, 153, Tikhookeanskaya st., 680042, Khabarovsk, Russia \\ ${ }^{2}$ Khabarovsk Department, Institute of Applied Mathematics, 54, Dzerzhinskogo st., 680000, Khabarovsk, Russia \\ ${ }^{3}$ South-West State University, 94, 50 let Oktyabrya st., 305040 Kursk, Russia \\ vzavod@mail.ru, o_garok@rambler.ru, apk3527@mail.ru
}

PACS 61.48.De, 82.70.Dd

DOI 10.17586/2220-8054-2017-8-5-635-640

\begin{abstract}
Modeling approaches based on the density functional theory (DFT): the Kohn-Sham (KS) method and orbital-free (OF) method are used to for calculation of the binding energies per atom as functions of the diameter of single-wall carbon nanotubes (SWCNTs) with the open ends. It is shown that this energy has a minimum at a diameter of about $1.1-1.2 \mathrm{~nm}$. The experiments made by means of Raman spectroscopy have shown that diameters of SWCNTs mainly lie in the range of $1-1.5 \mathrm{~nm}$.
\end{abstract}

Keywords: SWCNT, energy, diameter, modeling, Kohn-Sham, orbital free, Raman spectroscopy.

Received: 2 October 2017

Revised: 4 October 2017

\section{Introduction}

Single-wall carbon nanotubes (SWCNTs), discovered at the end of the last century are at the center of attention of many research groups. Many of those researchers are interested in the atomic and electronic structures of SWCNTs, their mechanical properties, interaction with other materials and among themselves, and many other things. However, despite huge number of the works devoted to the study of UNT, some of their main features are still obscure. In particular, one of main questions remains without answer: are the diameters of the tubes defined? Most often it is simply specified in literature that diameter of SWCNTs lies within $0.7-1.6$ nanometers. At the same time there are data that it is possible to receive SWCNTs with the minimum diameter of 0.3 nanometers [1] and maximum of 12 nanometers [2]. Experimental distributions of SWCNTs on diameter values with maxima in the range from 1 to 1.5 nanometers are given in $[3,4]$. Energy dependence of the armchair tubes on their diameters is obtained in [5] by the semi-empirical PM3 method of computer modeling; the energy minimum is revealed for a diameter of about 0.8 nanometers.

We did not find other data regarding the dependence of the SWCNTs' energy on the diameter value, therefore we performed our own calculations from the first principles, using two methods based on DFT [6]: namely, the KS [7] method realized in the FHI96md [8] package and the orbital-free (OF) method described in [9-12].

\section{Methods and models used for modeling}

The KS method is widely known and does not need a detailed discussion here. It is enough to remind that in this approach, the system of the one-electron equations of Schrödinger is replaced with a set of the Kohn-Sham equations in which each electron interacts with total density $\rho(\boldsymbol{r})$, formed by all electrons:

$$
-\frac{\hbar^{2}}{2 m} \Delta \psi_{i}(\boldsymbol{r})+V_{e f f}(\boldsymbol{r}) \psi_{i}(\boldsymbol{r})=\varepsilon_{i} \psi_{i}(\boldsymbol{r})
$$

where

$$
V_{e f f}(\boldsymbol{r})=\int \frac{\rho\left(\boldsymbol{r}^{\prime}\right)}{\left|\boldsymbol{r}^{\prime}-\boldsymbol{r}\right|} d \boldsymbol{r}^{\prime}+V_{x c}(\boldsymbol{r}), \quad \rho_{e}(\boldsymbol{r})=\sum_{i=1}^{N_{e}}\left|\psi_{i}(\boldsymbol{r})\right|^{2},
$$

$V_{x c}(\boldsymbol{r})$ is the exchange-correlation potential which can be calculated in some approach, $\psi_{i}$ is the wave function corresponding to the $i$-th one-electron state, $\varepsilon_{i}$ is the energy of this state.

The OF approach does not operate with wave functions (orbitals), but with the total electron density only. Actually, this approach is consecutive development of the hypothesis of Hohenberg and Kohn [6], that the ground state of a quantum system can be described by means of only electronic density. Thus, the total energy of the system can be found by minimization of a certain functional:

$$
E[\rho]=\int \varepsilon(\rho) d \boldsymbol{r}=\int V(\boldsymbol{r}) \rho(\boldsymbol{r}) d \boldsymbol{r}+\frac{1}{2} \int \varphi(\rho) \rho(\boldsymbol{r}) d \boldsymbol{r}+\int \varepsilon^{e x-c}(\rho) d \boldsymbol{r}+\int \varepsilon^{k i n}(\rho) d \boldsymbol{r},
$$


where $\varepsilon^{e x-c}$ and $\varepsilon^{k i n}$ are the exchange-correlation and kinetic energy functionals.

A basis of the OF approach is finding of the density $\rho(\boldsymbol{r})$ turning into zero the functional $F[\rho]$, which is a variation derivative of the functional of energy (1):

$$
F[\rho] \equiv \frac{\delta \varepsilon[\rho]}{\delta \rho}=V(\boldsymbol{r})+\varphi(\boldsymbol{r})+\mu_{k i n}(\rho)+\mu_{e x-c}(\rho)=0,
$$

where the density $\rho(\boldsymbol{r})$ must satisfy the condition $\int \rho(\boldsymbol{r}) d \boldsymbol{r}=N(N$ is the number of electrons in the system); the potentials $\mu_{k i n}(\rho)$ and $\mu_{e x-c}(\rho)$ are the variation derivatives $\mu_{k i n}(\rho)=\frac{\delta \varepsilon_{k i n}(\rho)}{\delta \rho}, \mu_{e x-c}(\rho)=\frac{\delta \varepsilon_{e x-c}(\rho)}{\delta \rho}$. If $\varepsilon^{k i n}$ is known, equation (3) may be solved by any iteration method, and then the total energy may be calculated.

The OF approach gained noticeable development in the last 20 years (see for example [13-18]), however its supporters have not yet achieved serious success. In our opinion, the reason for difficulties is that their works rely on the authority of Hohenberg and Kohn, who declared that there is a universal functional of kinetic energy and the task consists only in that to find it.

However, as shown recently [19,20], the idea of Hohenberg and Kohn about the existence of a universal density functional leading to the energy minimum is not strictly proved. Thus, there are bases to believe that attempts to construct an OF method based on use of any universal functional are obviously doomed to failure.

The OF method, based on the kinetic energy functionals $\varepsilon^{k i n}$ specified for the each type of atoms was developed in our previous works [9-12]. We demonstrated ability of our method to describe the structure and energy of atomic systems consisting of two, three, and four atoms of various types. In the present work, we use this method for more large systems: namely, for simulation of carbon nanotubes.

Taking into account the restrictions by the sizes of atomic systems which are available for the KS method and for observance of uniformity of calculations, we limited ourselves to consideration of nanotubes fragments of the armchair type in the form of rings consisted of the four atomic chains. Diameters of rings were varied from 0.41 nanometers to 1.55 nanometers. Thus, the number of the atoms included into the studied system changed from 48 to 176. The kinetic energy functional for carbon atoms were taken the same like in [10]. The cell sizes for calculations by the OF method were taken $40 \times 40 \times 40$ a.e. $^{3}$ with splitting $150 \times 150 \times 150(1$ a.u. $=0.529 \AA)$. For calculations by the KS method, we used a cell of the same size; the cutting energy for the set of plane waves was equal 40 Rydberg; exchange and correlation interactions were calculated in both cases in the LDA approach [21]. In all cases, atoms of carbon could move under the influence of interatomic forces and find the equilibrium positions during of calculations.

\section{Results of modeling}

First of all, we compared interatomic distances $d$ obtained in our calculations with literature data. Results are collected in Table 1.

TABLE 1. Interatomic distances $d$ obtained by us for fragments of open SWCNTs compared with other data

\begin{tabular}{|c|c|c|c|c|}
\hline Source & OF calculation & KS calculation & Other calculations & Experiment \\
\hline \hline$d$, inside, $\AA$ & 1.42 & 1.39 & $1.42[22]$ & $1.42[23]$ \\
\hline$d$, edge, $\AA$ & 1.32 & 1.28 & & \\
\hline
\end{tabular}

Our calculations give the sizes of distances between internal atoms of SWCNTs close to the literary data. Unfortunately, we did not find works in which experimental or theoretical data on interatomic distances at open edges of SWCNTs are provided. However in [24,25] it is shown by modeling by various methods that in carbon systems of diamond type the distance between edge atoms decrease to $1.35-1.38 \AA$ against $1.55 \AA$ in the central part. Qualitatively it coordinates with our results about reduction of interatomic distance on the ends of nanotubes in comparison with distances between internal atoms.

In Fig. 1, the binding energies (per atom) are presented for the considered SWCNT fragments. First, we see that the values received by the OF method (the curve 1) and by the KS method (the curve 2) are well coordinated with each other, differing less than for 10 percent. Both methods show the existence of a minimum of energy at similar values of diameter: $D_{\min }=1.1$ nanometer for the OF method, and $D_{\min }=1.24$ nanometer for the KS method. For comparison, the semi-empirical PM3 method data [4] are given in the same Fig. 1 (the curve 4). 
These data show a minimum at $D_{\min }=0.8$ nanometer. In our opinion, this minimum is not very reliable as it is presented by only one point which obviously drops out of the values next to it.

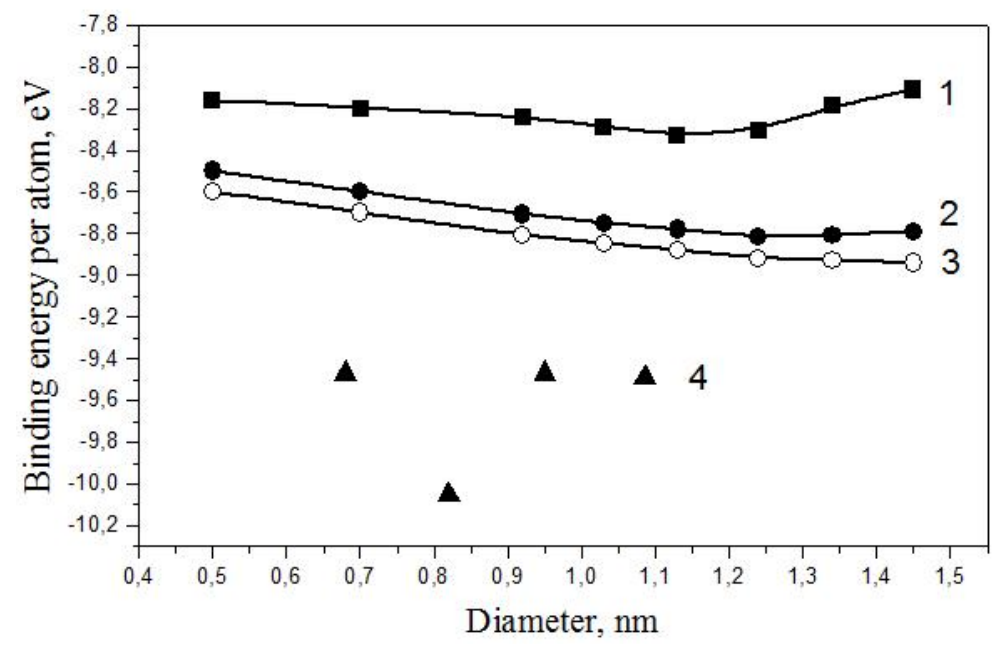

FIG. 1. The binding energy $v s$ diameter of SWCNT. 1 - OF calculations; 2 - KS calculations with open ends; 3 - KS calculations of infinite tubes; 4 - PM3 calculations [4]

There is the remaining question: what is the physical reason for the emergence of a minimum of the atomic energy while diameter of a tube increases? Two counteracting mechanisms of energy change are necessary for the emergence of a minimum. The first mechanism is obvious: the total number of the system's atoms increases with increase of diameter. As a rule, it conducts to increasing of binding energy if is only not followed by specific reorganizations of atomic structure, like, for example, it is happened in "magic" clusters. In our case, the structure of the system does not change therefore the binding energy has to grow on the absolute value with increase of diameter. Besides, the increase in diameter automatically is followed by reduction of curvature of a tube that in addition lowers the energy of the system as it approaches to the energy of graphene. However in the case of SWCNTs of finite sizes having the open ends there is one more possible mechanism of the energy changing; this is the interaction of the atoms which are on the ends of a tube, among themselves. The greater the diameter, the greater the average distance between these atoms, and correspondingly, the lower their contribution to the system's total energy.

To verify this hypothesis, we carried out additional calculations by the KS method, using the fact that the FHI96md package, which we applied to calculations, is adapted for periodic calculations and at an appropriate choice of parameters of the supercell can model an infinite nanotube (i.e. not having the ends). In this case it appeared that the binding energy falling on one atom has no minimum, and monotonously grows on an absolute value (the curve 3). That is, interaction of the edge atoms really responsible for the energy minimum.

\section{Technique of experiment}

The colloidal solutions (CSs) with multi-walled carbon nanotubes (MWCNTs) (Nanocyl production, Belgium), synthesized by CCVD method with nanocatalysts on the basis of $\mathrm{CoO}$, were used as a basis of investigation. Functionalization of MWCNTs was reached by hashing within 5 hours in an ultrasonic bathtub in the acid environment $\left(\mathrm{H}_{2} \mathrm{SO}_{4}\right.$ and $\mathrm{HNO}_{3}$ in the ratio 3:1) with a warming up no more than $42{ }^{\circ} \mathrm{C}$. Deionized water was added to solution after cooling in the equilibrium ratio; than it was filtering, washing, and the subsequent drying was carried out like [26]. The level of functionalization of MWCNTs was determined by use of the FT-IR spectra (Nicolet iS50, $\left.0.125 \mathrm{~cm}^{-1}\right)$.

The produced CSs were put as drops within the interelectrode space (100, 500, 1000 and $1500 \mu \mathrm{m}$ of width) of the film chip made like [26]. The constant voltage (varied from 10 to $50 \mathrm{~V}$ ) was applied to electrodes.

After evaporation of the drop some structures were formed in the electric field (Fig. 2). It is conditionally possible to divide them to fractals (with fractal dimensionality of $\left.D_{F R}=1.7-2.2\right)$, fractal clusters $\left(D_{F R}=1.6-\right.$ 1.8) consisting, probably, of very small nanoparticles, and piecewise linear (threadlike) structures. According to SEM and ASM data, the simultaneous formation of both fractal clusters, and piecewise linear structures was observed in case of change of voltage from 20 to $45 \mathrm{~V}$. 


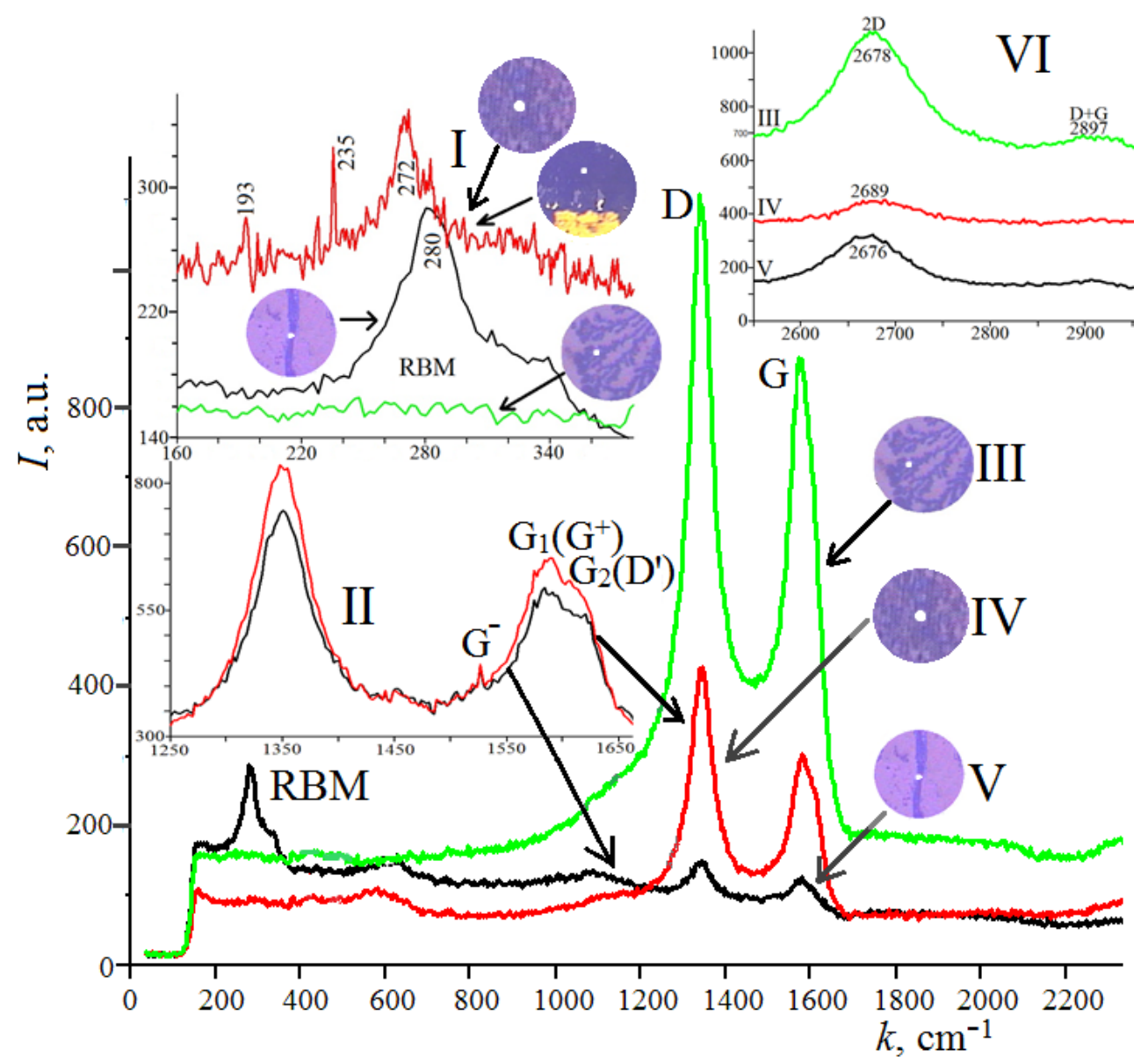

FIG. 2. Combinational Raman spectra (CRS) on the piecewise linear structures (V), on the fractal clusters (IV), and on the fractal structures (III) formed from MWCNT; CRS in the RBM area with pictures of structures (I); CRS in the area of lines $G^{-}, G_{1}\left(G^{+}\right)$, and $G_{2}\left(D^{\prime}\right)$ (II); CRS at frequencies higher than $2200 \mathrm{~cm}^{-1}$ (VI) for the cases III, IV, and V

In the combinational Raman spectra (CRS) (OmegaScope ${ }^{T M}$ the Raman microspectrometer, $\left.532 \mathrm{~nm}, 0.8 \mathrm{~cm}^{-1}\right)$, lines $D=1336-1353 \mathrm{~cm}^{-1}$ and $G=1567-1600 \mathrm{~cm}^{-1}$, have been found (Fig. 3) (both with electric field, and without the field), which are typical for MWCNTs. However, in the low-frequency area of CLS so-called radial breath modes (RBM) have been found in the range of $100-360 \mathrm{~cm}^{-1}$, typical only for SWCNTs [27] (see the insertion $I$ in Fig. 3). RBM lines were observed for the particles formed directly on electrodes (193, 235 and $\left.272 \mathrm{~cm}^{-1}\right)$ and also for the fractal clusters and the piecewise linear structures $\left(280 \mathrm{~cm}^{-1}\right)$ whereas they were absent for fractal structures (see the insertion $I$ in Fig. 3). It directly indicates the perpendicular orientation of the SWCNTs to a substrate. Values of diameters of SWCNTs calculated as $d=285 \mathrm{~cm}^{-1} / \omega$ [27]: for the piecewise linear structures $d \approx 1 \mathrm{~nm}$, and $d=1-1.5 \mathrm{~nm}$ for the fractal clusters and for SWCNTs formed on the electrodes.

\section{Conclusion}

The calculations which are carried out by two methods based on DFT, namely the Kohn-Sham method and the orbital-free method, showed that the binding energy of carbon nanotubes has an energy minimum with a diameter of tube about $1.1-1.2$ nanometers. It is revealed that interaction of atoms which are on the open ends of tubes is responsible for the emergence of an energy minimum. The experimental study, which was carried out by means of Raman spectroscopy has shown that SWCNTs which are formed in contact with multiwall carbon nanotubes have diameters in the range of $1-1.5$ nanometers irrespective of presence of electric voltage. 


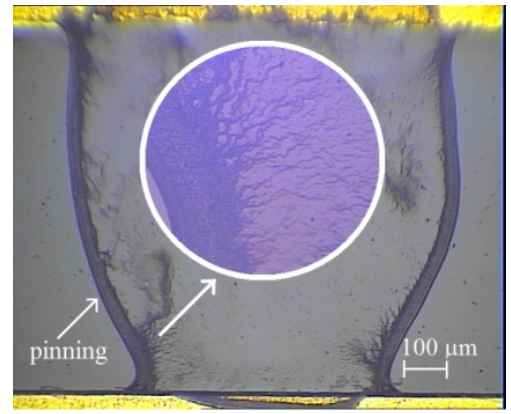

(a)

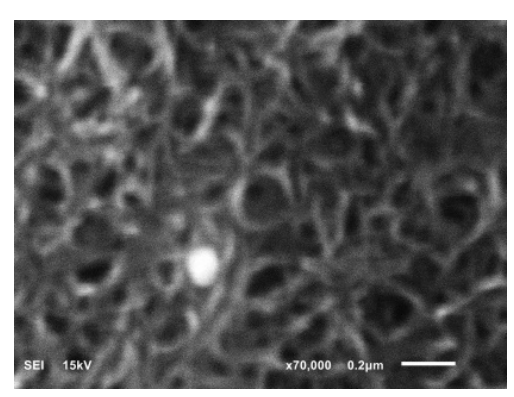

(d)

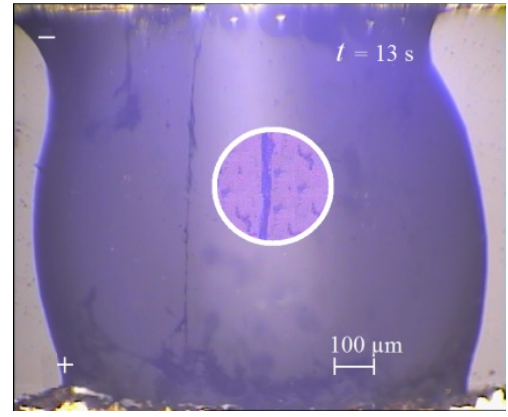

(b)

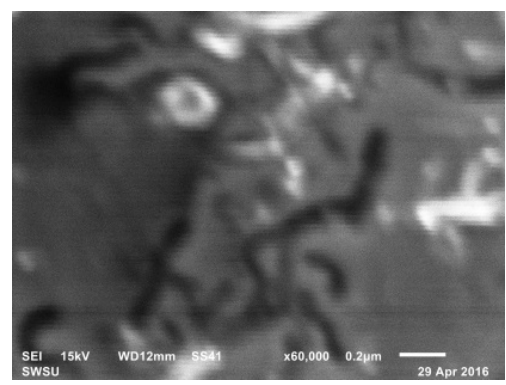

(e)

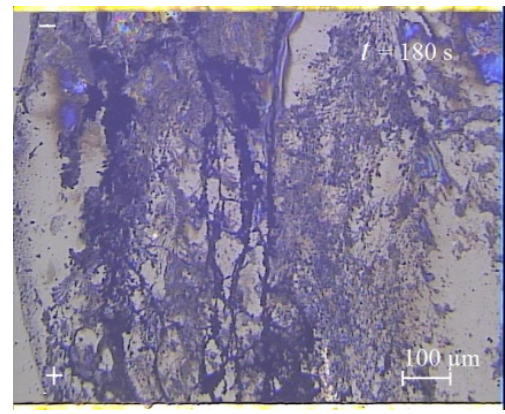

(c)

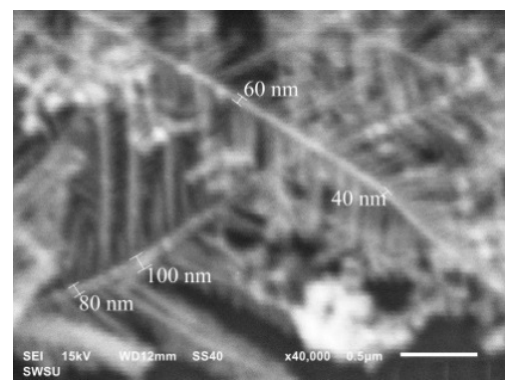

(f)

FIG. 3. Confocal and SEM images on polycrystalline glass: MWCNTs without voltage (a, d), piecewise linear structures (b), fractal clusters and diffusion structures at $U=21 \mathrm{~V}$ (c), and SEM images of MWCNTs at $U=20 \mathrm{~V}$ (e and f) with the similar structures having dark and light contrasts

\section{Acknowledgements}

This work is performed with financial support of the Ministry of Education and Science of the Russian Federation within the task No. 16.2814.2017/PCh. One of authors (A.P. Kuz'menko) expresses gratitude to employees of the South-West State University (Kursk, Russia) A. E. Kuzko, Thet Phyo Naing, and Myo Min Than.

\section{References}

[1] Lee K., Chong Rae Park. Effects of chirality and diameter of single-walled carbon nanotubes on their structural stability and solubility parameters. Royal Society of Chemistry Advances, 2014, 4, P. 33578-33581.

[2] Chin Li Cheung, Andrea Kurtz, Hongkun Park, Charles M. Lieber. Diameter-Controlled Synthesis of Carbon Nanotubes. J. Phys. Chem. $B, 2002$, 106, P. 2429-2433.

[3] Eletskiy A.V. Carbon nanotubes. Uspekhi Fizicheskikh Nauk, 1997, 167 (9), P. 945-972 (in Russian).

[4] Kiang C.-H., Goddard III W.A., Beyers R., Bethune D.S. Carbon nanotubes with single-layer walls. Carbon, 33 (7), P. 903-914.

[5] Boguslavskaya E.S., Bazhin I.V. Mechanical properties of carbon nanotubes and of their contacts. Fazovye perekhody, uporyadochennye sostoyaniya i novye materialy, 2009, 11, 4 p. (in Russian).

[6] Hohenberg H., Kohn W. Inhomogeneous electron gas. Phys. Rev. B, 1964, 136, P. 864-871.

[7] Kohn W., Sham J.L. Self-consistent equations including exchange and correlation effects. Phys. Rev. A, 1965, 140, P. 1133-1138.

[8] Beckstedte M., Kley A., Neugebauer J., Scheffler M. Density functional theory calculations for poly-atomic systems: electronic structure, static and elastic properties and ab initio molecular dynamics. Comp. Phys. Commun., 1997, 107, P. 187-205.

[9] Zavodinsky V.G., Gorkusha O.A. A simple quantum-mechanics way to simulate nanoparticles and nanosystems without calculation of wave functions. International Scholarly Research Network Nanomaterials, 2012, 531965, 3 p.

[10] Zavodinsky V.G., Gorkusha O.A. Quantum-Mechanical Modeling without Wave Functions. Physics of the Solid States, 2014, 56 (11), P. 2329-2335.

[11] Zavodinsky V.G., Gorkusha O.A. Development of an orbital free approach for simulation of multiatomic nanosystems with covalent bonds. Nanosystems: Physics, Chemistry, Mathematics, 2016, 7 (3), P. 427-432.

[12] Zavodinsky V.G., Gorkusha O.A. Development of the orbital free approach for heteroatomic systems. Nanosystems: Physics, Chemistry, Mathematics, 2016, 7 (6), P. 1010-1016.

[13] Wang Y.A., Carter E.A. Theoretical Methods in Condensed Phase Chemistry. In Progress in Theoretical Chemistry and Physics, Kluwer, Dordrecht, 2000, P. 117-184. 
[14] Huajie Chen, Aihui Zhou. Orbital-free density functional theory for molecular structure calculations. Numerical Mathematics: Theory, Methods and Applications, 2008, 1 (1), P. 1-28.

[15] Karasiev V.V., Jones R.S., Trickey S.B., Harris F.E. Recent advances in developing orbital-free kinetic energy functionals. In New Developments in Quantum Chemistry, 2009, P. 25-54.

[16] Chakraborty D. Computing the kinetic energy from electron distribution functions. Ph.D. (Chemistry) Thesis, McMaster University, Hamilton, Ontario, Canada, 2011.

[17] Huang C., Carter E.A. Nonlocal orbital-free kinetic energy density functional for semiconductors. Phys. Rev. B, 2010, 81, 045206-15.

[18] Ho G.S., Ligneres V.L., Carter E.A. Introducing PROFESS: A new program for orbital-free density functional theory calculations. Comp. Phys. Commun., 2008, 179, P. 839-854.

[19] Sarry A.M., Sarry M.F. On the density functional theory. Fizika Tverdogo Tela, 2012, 54 (6), P. 1237-1243 (in Russian).

[20] Bobrov V.B., Trigger S.A. The problem of the universal density functional and the density matrix functional theory. J. Exper. Theor. Phys., 2013, 116 (4), P. 635-640.

[21] Perdew J.P., Wang Y.A. Accurate and simple density functional for the electronic exchange energy. Phys. Rev. B, 1986, 33, P. 8800-8802.

[22] Huhtala M., Kuronen A., Kimmo Kaski. Computational studies of carbon nanotube structures. Computer Physics Communications, 2002, 147, P. 91-96.

[23] Danilov C.V. Simulation of atomic structure and the X-Ray structure analysis of carbon nanotubes. PhD Thesis, Petrozavodsk State University, Petrozavodsk, Russia, 2013 (in Russian).

[24] Barnard A.S., Russo S.P., Snook I.K. Surface structure of cubic diamond nanowires. Surf. Sci., 2003, 538, P. $204-210$.

[25] Cheng Yang, H. Chuan Kang. Cluster study of the dimer geometry on the C(100) surface. Surf. Sci., 1998, 409, P. 521-527.

[26] Kuzmenko A.P., Thet Phyo Naing, et al. Self-assembly and Self-organization Processes of Carbon Nanotubes in the Colloidal Systems. $J$. of Nano- and Electronic Physics, 2015, 7 (4), P. 04014(3).

[27] Thomsen C., Reich S. Raman scattering in carbon nanotubes. Topics Appl. Physics, 2007, 108, P. $115-232$. 